\title{
Characterization of Proton Irradiation Effect in Semiconductor Material
}

\author{
Haider F. Abdul Amir and Fuei Pien Chee, Member, IACSIT
}

\begin{abstract}
The different radiation environments either in space, high energy physics experiments, nuclear environment or fabrication process as well as for standard terrestrial operation possess an impact on the semiconductor devices. This makes that the devices based on III-V semiconductors are probable to be critical components of future electronic systems as the demand for greater robustness and susceptibility to well function in rigorous radiation environments continue to increase. In this paper, the penetration of proton, utilizing ion Hydrogen $\left(\mathrm{H}^{+}\right)$into Silicon $(\mathrm{Si})$ materials is being simulated using the program simulation SRIM (stopping and range of ions in matter). By using the SRIM simulation program, the final 3D distribution of the ions and all kinetic phenomena associated with the ion's energy loss: target damage, sputtering, ionization, and phonon production can be calculated. From the findings, it is observed that the penetration of $\mathrm{H}^{+}$ions into the target $\mathrm{Si}$ layer leads to production of lattice defects in the form of vacancies, defect clusters and dislocations. The simulation results also show that the level of degradation in the $\mathrm{Si}$ materials is highly depending on the projected range, the nuclear stopping and electronic stopping of $\mathrm{H}^{+}$ions. There is no apparent difference in the radiation damage when the incident angle of $\mathrm{H}^{+}$ions is varying.
\end{abstract}

Index Terms-Hydrogen, silicon, lattice defects.

\section{INTRODUCTION}

Studies for introduction of atoms into a solid substrate by bombardment of the solid with ions in the electron-volt $(\mathrm{eV})$ to mega-electron-volt $(\mathrm{MeV})$ energy range have always received great interest. These interests have been stimulated by the feasibilities of synthesizing novel materials with potential applications in the semiconductor, mineral processing and metallurgy, corrosion and optical fields. The physical properties for a particular solid substrate are always sensitive to the presence of a trace amount of foreign atoms. Mechanical, electrical, optical, magnetic and superconducting properties are all affected and sometimes may even be dominated by the existence of such foreign atoms [1].

The main sources of the high energy charged particles in space environments are from the trapped radiation in the magnetosphere and Van Allen belts, galactic cosmic rays (GCRs) and solar particle events (SPEs) [2]. Protons are the major component of Van Allen belts and also the largest component of the cosmic rays with a composition of about $92 \%$. Proton is a subatomic particle with an electric charge of

Manuscript received June 15, 2013; revised August 25, 2013. This work was supported in part by the Malaysia Science Fund 2007, MOSTI, Project No.: SCF0046-STS-2007, with title "Effects of Total Dose Irradiation on Semiconductor Devices".

The authors are with the School of Science and Technology, University of Malaysia Sabah, Kota Kinabalu, Malaysia (e-mail: haider@ums.edu.my; fpchee06@ieee.org).
+1 and is composed of three fundamental particles: two up quarks and one down quark.

Interaction of radiation with semiconductor devices leads to the deposition of energy which can be either a transient or permanent effect [3]. These effects can be broadly categorized into Electromagnetic Pulse (EMP) effects, high dose rate effects, Single Event Effects (SEEs) and total dose effects. The radiation damage due to a single energetic particle, however, normally gives arise to SEEs which can be classified to three effects:

1) Single Event Upset (SEU) or soft error is defined as radiation-induced errors in semiconductor devices caused when charged particles lose energy by ionizing the medium through which they pass, leaving behind a wake of electron-hole pairs. An SEU may occur in analog, digital, or optical components, or may have effects in surrounding interface circuitry [4]. SEUs typically appear as transient pluses in logic or support circuitry, or as bit flips in memory cells or registers. SEU may cause data corruption and alter program depending on the location of the upset. A reset or rewriting of the device results in normal device behavior thereafter [5]. The SEU itself is not considered permanently damaging to the transistor's or circuits' functionality unlike the case of single event latch-up (SEL), single event gate rupture (SEGR), or single event burnout (SEB).

2) SEL is a potentially destructive condition involving parasitic circuit elements forming silicon controlled rectifier (SCR). This may cause loss of device functionality due to a single-event induced current state. SELs are hard errors, and are potentially destructive [5]. The SEL results in a high operating current, above device specifications. The latched condition can destroy the device, drag down the bus voltage, or damage the power supply.

3) SEB is a condition that can cause device destruction due to a high current state in a power transistor. This event may occur when the passage of a single heavy ion forward biases the thin body region under the source of the device. If the drain-to-source voltage of the device exceeds the local breakdown voltage of the parasitic bipolar, the device can burn out due to large currents and high local power dissipation. SEB's effects also include gate rupture, frozen bits, and noise. SEB susceptibility has been shown to decrease with increasing temperature.

In this paper, the simulation of damage induced to silicon by $\mathrm{H}^{+}$ions using SRIM-TRIM software will be presented. This is because delicate electronic circuits can be highly vulnerable to energetic protons. Silicon is being discussed as it is the dominant semiconductor material in the electronic industry. Silicon can retain its semiconductor characteristics even at a higher temperature while germanium can't [6]. 
Besides, silicon's native oxide can be easily grown in a furnace and it forms a better semiconductor or dielectric interface than any other material.

\section{EXPERIMENTAL DETAILS}

The target utilized in the simulation, Si has a band gap energy of $E_{g}=1.12 \mathrm{eV}$ at room temperature with an atomic number of 14 . The density of $\mathrm{Si}$ is $2.3290 \mathrm{~g} / \mathrm{cm}^{3}$ with electron configuration of $3 \mathrm{~s}^{2} 3 \mathrm{p}^{2}$.

SRIM is a computer simulation that uses Monte Carlo method and it contains TRIM calculation. It is applicable in ion- solid interactions and has a number of distinct advantages over analytical calculations based on transport theory. The Monte Carlo method requires averaging over many simulated trajectories particles and relies on the binary collision model.

The SRIM-TRIM calculates the range of ions in matter using collisions of ions-atoms. It also calculates the $3 \mathrm{D}$ spreads of ions as well as all the kinetic phenomena that are related with the loss of energy: damage of the target, sputtering, ionization and phonon production. The TRIM calculation runs even it is interrupted awhile and the output results can be saved and used later. The results obtain in this simulation have shown excellent agreement with a wide range of experimental data for ions with energies below 1 $\mathrm{MeV}$.

In this research, the damage induced by $\mathrm{H}+$ ions on $\mathrm{Si}$ layer is simulated. The energies for the incident ions are varying from $10 \mathrm{keV}$ up to $1 \mathrm{MeV}$. The collision event of 1000 ions is simulated at different incident angle of $0^{\circ}, 30^{\circ}$ and $60^{\circ}$.

By the simulation of detailed calculation with full damage cascades, the plots of ion trajectories, depth vs. Y-Axis, depth vs. Z-Axis, transverse view, ionization, phonons, collision events, atom distributions and energy to recoil can be obtained.

\section{RESULTS AND DISCUSSIONS}

When the $\mathrm{H}^{+}$ion slows down and comes to rest in the $\mathrm{Si}$ crystal, a number of collisions are made with the lattice atoms In these collisions, $\mathrm{H}^{+}$ion will loses its energy at a rate of $d E / d x$ of a few 100 electrons - volts per nanometer, depending on its incident energy [4]. The range $R$ can be determined by the rate of energy loss along the path of the ion,

$$
R=\int_{E_{H}}^{0} \frac{1}{d E / d x} d E
$$

$E_{H}=$ is the incident energy of the $\mathrm{H}^{+}$ion as it penetrates the solid.

$R$ is defined as the total distance that the projectile travels in coming to rest. However, in most of the conditions and applications, the projected range, $R_{p}$, is the quantity of interest. $R_{p}$ is the total path of length of the projectile measured along the direction of incidence where

$$
R_{p} \cong \frac{R}{1=\left(M_{2} / 3 M_{1}\right)}
$$

where $M_{1}$ is the atomic mass of $\mathrm{H}^{+}$ion $(1 \mathrm{amu})$ and $M_{2}$ is the atomic mass of $\mathrm{Si}(28 \mathrm{amu})$ in this case. The projected range of $\mathrm{H}^{+}$ion in energy range from $10 \mathrm{keV}$ to $1000 \mathrm{keV}$ is as shown in the plotting of Fig. 1.

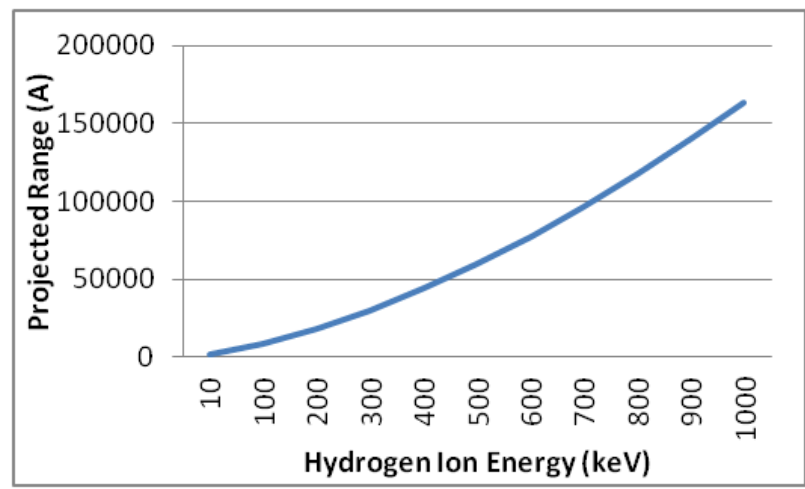

Fig. 1. Projected range, $R_{p}$ of $\mathrm{H}^{+}$ion in Silicon (Si).

The energy loss, $d E / d x$ of $\mathrm{H}^{+}$ion in $\mathrm{Si}$ can be divided into two parts: the energy transferred by the $\mathrm{H}^{+}$ion to the $\mathrm{Si}$ electrons (called electronic stopping or inelastic energy loss) and to the Si nuclei (called nuclear stopping or elastic energy loss). The nuclear stopping and electronic stopping energy loss of $\mathrm{H}^{+}$ion in $\mathrm{Si}$ at different energy level are as shown in Table I. It is found that the electron energy loss is always higher than the nuclear energy loss.

TABLE I:THE ELECTRONIC STOPPING AND NUCLEAR STOPPING ENERGY LOSS OF H+ ION IN SI

\begin{tabular}{|c|c|c|}
\hline $\begin{array}{c}\text { Ion Energy } \\
(\mathrm{keV})\end{array}$ & $\begin{array}{c}\mathrm{dE} / \mathrm{dx} \text { Electron } \\
(\mathrm{keV} / \mu \mathrm{m})\end{array}$ & $\begin{array}{c}\mathrm{dE} / \mathrm{dx} \text { Nuclear } \\
(\mathrm{kev} / \mu \mathrm{m})\end{array}$ \\
\hline 10 & $7.27 \mathrm{E}+01$ & $9.80 \mathrm{E}-01$ \\
\hline 100 & $1.15 \mathrm{E}+02$ & $2.01 \mathrm{E}-01$ \\
\hline 200 & $9.12 \mathrm{E}+01$ & $1.17 \mathrm{E}-01$ \\
\hline 300 & $7.63 \mathrm{E}+01$ & $8.41 \mathrm{E}-02$ \\
\hline 400 & $6.64 \mathrm{E}+01$ & $6.64 \mathrm{E}-02$ \\
\hline 500 & $5.93 \mathrm{E}+01$ & $5.52 \mathrm{E}-02$ \\
\hline 600 & $5.39 \mathrm{E}+01$ & $4.74 \mathrm{E}-02$ \\
\hline 700 & $4.96 \mathrm{E}+01$ & $4.17 \mathrm{E}-02$ \\
\hline 800 & $4.61 \mathrm{E}+01$ & $3.72 \mathrm{E}-02$ \\
\hline 900 & $4.32 \mathrm{E}+01$ & $3.37 \mathrm{E}-02$ \\
\hline 1000 & $4.07 \mathrm{E}+01$ & $3.08 \mathrm{E}-02$ \\
\hline
\end{tabular}

The electron energy loss is due to the following mechanisms:

1) Direct kinetic energy transfers to $\mathrm{Si}$ electrons which is mainly caused by the electron-electron collisions

2) Excitation or ionization of target atoms,

3) Excitation of band or conduction electrons,

4) Excitation, ionization or electron capture of the projectile itself.

Fig. 2 and Fig. 3 show the $\mathrm{H}^{+}$ion track at $E_{H}=10 \mathrm{keV}$ and $E_{H}=1 \mathrm{MeV}$ with an incident angle of $0^{\circ}$ and $30^{\circ}$ respectively. The red dot in the ion track represents the vacancy created by the incident $\mathrm{H}^{+}$ion which means that an atom in the $\mathrm{Si}$ is displaced from its lattice site. The red dots show that the $\mathrm{H}^{+}$ ion create damage constantly. The clusters of green dots, namely recoil cascade, are the vacancies caused by the recoiling $\mathrm{Si}$ atoms. Subsequent ion irradiations lead to the overlaps of the ion trajectories and then develop a more uniform distribution of radiation damage. 


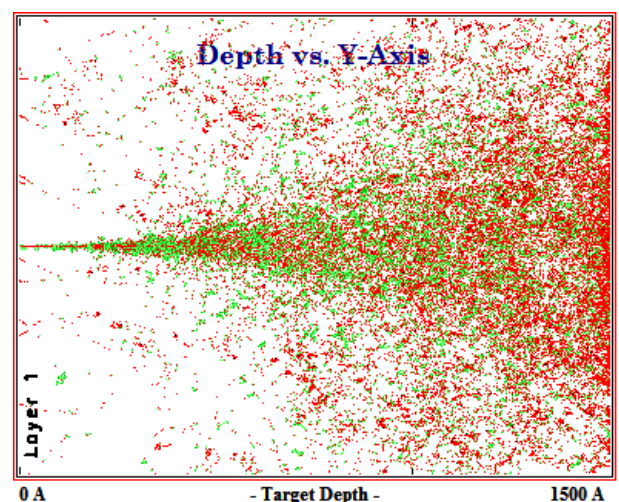

Fig. 2. The $\mathrm{H}^{+}$ion track at $\mathrm{Y}$-axis in $\mathrm{Si}$ at $E_{H}=10 \mathrm{keV}$ at incident angle of $0^{\circ}$.

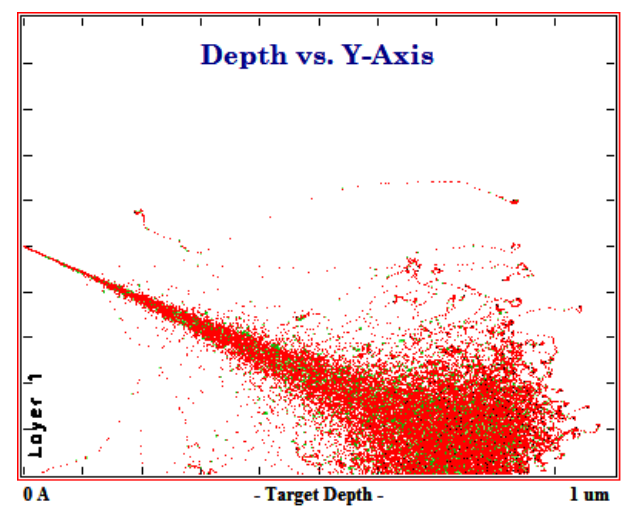

Fig. 3. The $\mathrm{H}+$ ion track at $\mathrm{Y}$-axis in $\mathrm{Si}$ at $E_{H}=100 \mathrm{keV}$ at incident angle of $30^{\circ}$.

TABLE II: INTERACTION OF H+ ION IN SI AT DIFFERENT ENERGY LEVEL AND INCIDENT ANGLE

\begin{tabular}{|c|c|c|c|c|c|}
\hline \multirow{2}{*}{$\begin{array}{c}\text { Ion Energy } \\
(\mathrm{keV})\end{array}$} & \multirow[t]{2}{*}{ Incident angle $\left({ }^{\circ}\right)$} & \multicolumn{3}{|c|}{ Damage Events } & \multirow{2}{*}{$\begin{array}{c}\text { Energy Absorbed by Si } \\
\text { (eV/ion) }\end{array}$} \\
\hline & & Total displacements/Ion & Total vacancies/Ion & Replacement collisions/Ion & \\
\hline \multirow[t]{3}{*}{10} & 0 & 9 & 8 & 1 & 214 \\
\hline & 30 & 10 & 9 & 1 & 230 \\
\hline & 60 & 10 & 9 & 1 & 231 \\
\hline \multirow[t]{2}{*}{100} & 0 & 20 & 19 & 1 & 517 \\
\hline & 60 & 19 & 18 & 1 & 484 \\
\hline \multirow[t]{3}{*}{1000} & 0 & 41 & 38 & 3 & 1140 \\
\hline & 30 & 41 & 38 & 3 & 1140 \\
\hline & 60 & 41 & 38 & 3 & 1140 \\
\hline
\end{tabular}

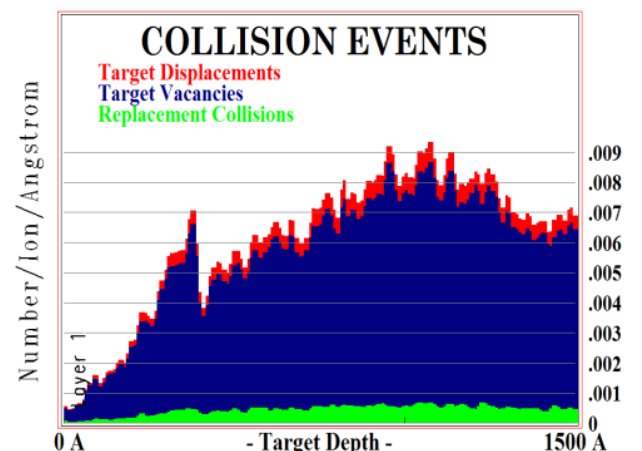

Fig. 4. The collision events in Si layer at $E_{H}=10 \mathrm{keV}$ at incident angle of $0^{\circ}$.

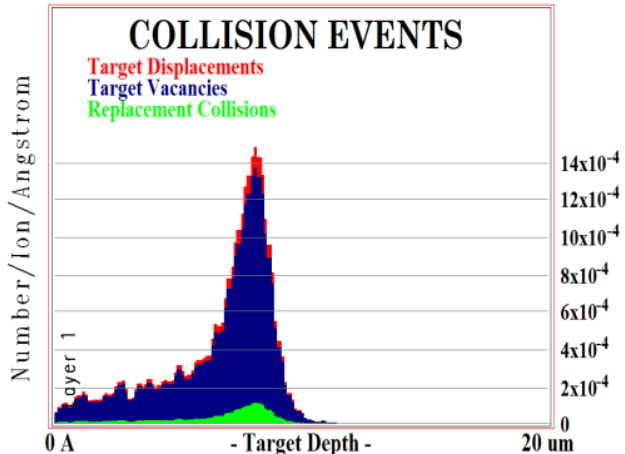

Fig. 5. The collision events in Si layer at $E_{H}=1 \mathrm{MeV}$ at incident angle of $0^{\circ}$.

During the collisions, the lattice atoms which are displaced by the incident $\mathrm{H}^{+}$ion are known as primary knock-on atoms (PKAs). The energy required to displace the lattice atom represents the displacement threshold and is called the displacement energy, $E_{d}$. The PKAs can in turn displace other atoms and ends up in creating a cascade of atomic collisions. This leads to the lattice disorder on the region around the ion track. The interaction of $\mathrm{H}^{+}$ion in $\mathrm{Si}$ at varying energy level and incident angle is as shown in Table II.
Fig. 4 and Fig. 5 show the number of target atom displacements per ion per Angstrom along the ion track at EH $=10 \mathrm{keV}$ and $\mathrm{EH}=1 \mathrm{MeV}$ respectively. The highest curve shows the target displacements while the lower curve is the target vacancies. This indicates that there are fewer vacancies than the displacement in this collision event. The lowest curve shows the replacement collision which indicates the number of incident $\mathrm{H}+$ ion that replaced the vacancy left by the recoiling $\mathrm{Si}$ atom.

Table III shows the simulation results for energy loss of $\mathrm{H}^{+}$ ion and recoil at different energy level and incident angle. Ionization refers to the energy loss to the target electrons. The data relating to 'Ions' is the direct energy transferred from the $\mathrm{H}^{+}$ion to Si while the data relating to the "Recoils" is energy transferred from recoiling $\mathrm{Si}$ atoms to their electrons.

TABLE III: ENERGY LOSS OF H+ ION AND RECOIL AT DIFFERENT ENERGY LEVEL AND INCIDENT ANGLE

\begin{tabular}{|c|c|c|c|c|c|}
\hline \multirow{3}{*}{$\begin{array}{c}\text { Ion Energy } \\
(\mathrm{keV})\end{array}$} & \multirow{2}{*}{ Incident angle $\left(^{\circ}\right)$} & \multicolumn{2}{|c|}{$\begin{array}{c}\text { \% of Energy Loss } \\
\text { in Ion }\end{array}$} & \multicolumn{2}{c|}{$\begin{array}{c}\text { of Energy Loss } \\
\text { in Recoil }\end{array}$} \\
\cline { 2 - 6 } & & 96.17 & 0.92 & 0.57 & 2.16 \\
\hline \multirow{3}{*}{10} & 0 & 95.93 & 1.01 & 0.59 & 2.29 \\
\cline { 2 - 6 } & 30 & 95.86 & 1.07 & 0.59 & 2.29 \\
\cline { 2 - 6 } & 60 & 99.22 & 0.16 & 0.14 & 0.45 \\
\hline \multirow{2}{*}{100} & 0 & 99.25 & 0.16 & 0.13 & 0.43 \\
\cline { 2 - 6 } & 30 & 99.25 & 0.16 & 0.13 & 0.43 \\
\cline { 2 - 6 } & 60 & 99.84 & 0.03 & 0.04 & 0.09 \\
\cline { 2 - 6 } & 0 & 99.84 & 0.03 & 0.04 & 0.09 \\
\cline { 2 - 6 } & 30 & 99.84 & 0.03 & 0.04 & 0.09 \\
\hline
\end{tabular}

\section{CONCLUSION}

The SRIM-TRIM simulation is crucial in the radiation testing of the semiconductor material as it describes the 
slowing down and scattering of energetic ion in solid well. From the simulation, $\mathrm{H}^{+}$ion is shown to impose dislocations and other types of electrical active defects in the Si layer. The deposition of energy by the incident $\mathrm{H}^{+}$ion in $\mathrm{Si}$ is not much effected by its incident angle.

\section{ACKNOWLEDGMENT}

The authors are thankful to Science Fund 2007, MOSTI, Project No.: SCF0046-STS-2007, with title "Effects of Total Dose Irradiation on Semiconductor Devices".

\section{REFERENCES}

[1] M. Nastasi, W. J. Mayer, and K. J. Hirnoven, Ion-solid Interaction Fundamentals and Applications, UK: Cambridge University Press, 2004.

[2] C. Claeys and E. Simoen, Radiation effects in advanced semiconductor materials and devices, Germany: Springer-Verlag Berlin Heidelberg, 2002.

[3] A.O. Guitièrrez, "Radiation Effects on Power MOSFETs," Ph.D's Dissertation. Rensselaer Polytechnic Institute, Troy, New York, May 1999.
[4] X. Li, K. Shen, C. M. Huang, and L. Chu, "A memory soft error measurement on production systems," 2007 USENIX Annual Technical Conference Proceedings, Santa Clara, CA, pp. 275-280, 2007.

[5] T. Z. Fullem, Radiation detection using single event upsets in memory chip, New York: State University of New York at Binghamton, 2006.

[6] A. D. Neaman, An Introduction to Semiconductor Devices, Singapore: McGraw-Hill, 2006.

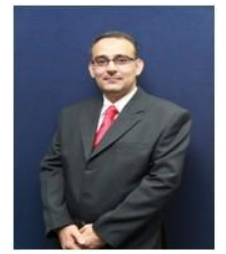

Haider F. Abdul Amir was born on $4^{\text {th }}$ June 1969 in Iraq. He obtained his Master and PHD degree in engineering physics (with Concentration on Semiconductor Devices, Instrumentations and Nuclear), from Faculty of Engineering, University of Gadjah Mada, Indonesia, secondment of Osaka University Japan. He had his first degree in Faculty of Sciences, University of Baghdad at Iraq. He had been an active researcher and lecturer at many universities and Institutions, in Iraq and Indonesia. He is currently working as Associate Professor in Universiti Malaysia Sabah (UMS), Malaysia since 2006. His current research interests are nanotechnology in electronic, semiconductor materials, nuclear physics and green technology. He has a track record of fundamental research on these topics which is documented by numerous publications. He has been reviewer for numerous journals and indexed papers, such as Borneo Science, IEEE, ScienceDirect. 